\title{
Désir de se sentir vivre et libre
}

Propos recueillis par Gilles Raveneau

Loïc Jean-Albert

chute libre, parachutiste

Loï Jean-Albert a vingt-huit ans. Il est marié et vient d'avoir un enfant. Parachutiste de haut niveau, professionnel depuis déjà dix ans, il a été aussi bien athlète qu'entraîneur, moniteur et instructeur, aussi bien plieur de parachute que concepteur, réparateur et vendeur. Il fait partie du conseil fédéral de la Fédération française de parachutisme en tant que représentant des professionnels et travailleurs indépendants. Il est par ailleurs pilote d'avion et breveté pilote-largueur. Il a tout pratiqué. À dix-huit ans, il intègre l'équipe de France de vol relatif à huit. De 1996 à 1999, il sera trois fois troisième au championnat du monde, trois fois second au championnat d'Europe et trois fois champion de France. Après cette série de victoires, il opte pour le free fly, dernière-née des disciplines du parachutisme, consistant à réaliser des figures acrobatiques en chutant à trois avec un vidéaste filmant la performance. Il y excelle tout autant, multipliant les bons résultats : champion de France (trois fois), d'Europe (deux fois), vainqueur de la coupe du monde en 2000 (troisième en 2001 et second en 2003). Il est également membre de l'équipe de France de sky surf, avec laquelle il se classera troisième au championnat du monde en 2003.

Depuis 1997, Loïc Jean-Albert pratique en outre assidûment le BASE-jump et la wing suit. Le BASE-jump consiste à s'élancer d'une falaise ou du sommet d'un gratte-ciel, et à n'ouvrir la voile qu'au dernier moment, à environ 150 mètres du sol. La wing suit est une combinaison-voile où le corps tient lieu d'armature et dont les ailes augmentent la capacité de planer. Elle permet ainsi d'améliorer le vol humain en réduisant de façon considérable la vitesse de chute verticale et en prolongeant d'autant la distance horizontale parcourue. Loïc Jean-Albert est d'ailleurs lui-même le concepteur de deux modèles de wing suit : S-Fly Expert et S-Fly Access.

Mes parents sont tous les deux parachutistes et ils pratiquaient bien avant ma naissance. Je suis né sur un aérodrome en quelque sorte... Toutes les fins de semaine, la famille était au centre de parachutisme. Cela fait vraiment partie de ma culture. J'ai commencé dès seize ans, c'est-à-dire l'âge minimum requis pour pratiquer. Mes frères s'y sont mis plus tard.

Au départ, le parachutisme était ma passion, puis c'est devenu mon métier. Le fait que ce soit à la fois une passion et un métier produit une différence dans la manière dont on aborde l'activité. Dans le rapport au risque, cela change forcément quelque chose. Lorsque l'on est professionnel, on est conduit à faire des choses que l'on ne ferait pas si c'était juste pour son plaisir. Il y a beaucoup de cascadeurs par exemple qui se sont fait avoir comme ça, parce qu'il fallait travailler. On doit être conscient de ce risque-là et ne pas se laisser piéger. Il faut être capable de dire : "Non, ça je ne le fais pas."

En adaptant ces comportements et le matériel utilisé, on peut faire du parachutisme de manière très sécurisée. Chaque pratiquant prend plus ou moins de risques selon ce qu'il recherche. La mesure du risque peut ainsi passer de un à cinquante. On ne peut pas dire: "Je fais du parachutisme et cela produit tel niveau de dangerosité. " Entre une activité loisir, qui se réalise en sautant d'un avion et en "ouvrant haut " un parachute "pépère ", et du BASE-jump dans des endroits engagés en montagne, il n'y a rien de commun. Il s'agit de parachutisme bien sûr, mais les disciplines et les modalités de pratique sont nombreuses ; les niveaux de risque et les marges de sécurité sont donc très différents.

Le risque est toujours présent. Il est accepté par les pratiquants. Il y a toujours un peu de fatalité en montagne, en mer, en aviation... Elle permet justement de se dégager de la focalisation sur ce qui fait peur sans être pour autant dangereux, et d'avoir les idées plus claires pour gérer l'action. La maitrise de soi tient principalement à la maitrise technique. Si l'on est sûr de sa technique, de la précision de ses trajectoires, on n'a pas d'appréhension. La maîtrise de soi se travaille. Un alpiniste, face à des situations dangereuses, sera bien plus lucide sur son premier saut en parachute qu'un pratiquant moyen. Son apprivoisement des situations dangereuses limitera sans doute la peur et n'inhibera pas son plaisir.

La peur apparaît quand on perd le contrôle. Je peux faire des choses engagées, mais tant que je contrôle la situation, que je sens où je vais, que je vois ce que je fais, je n'ai pas vraiment peur. Je ne suis pas à l'abri d'un incident technique, mais cela je l'accepte au départ. Les rares fois où j'ai eu peur, j'ai perdu le contrôle. Je sais que je suis dans une situation dangereuse et qu'il ne faut pas que «je me rate». Cela mapporte une certaine concentration sur ce que je vais faire. J'ai plus peur lorsqu'un gros chien est à côté de moi en train de grogner que lorsque je suis en haut d'une falaise pour un saut! Face au chien, je ne sais pas ce qui va se passer. La situation m'échappe, tout simplement; je ne contrôle pas. Si on compare cela à l'expérience de la drogue, des gens en ont besoin pour perdre le contrôle et le 


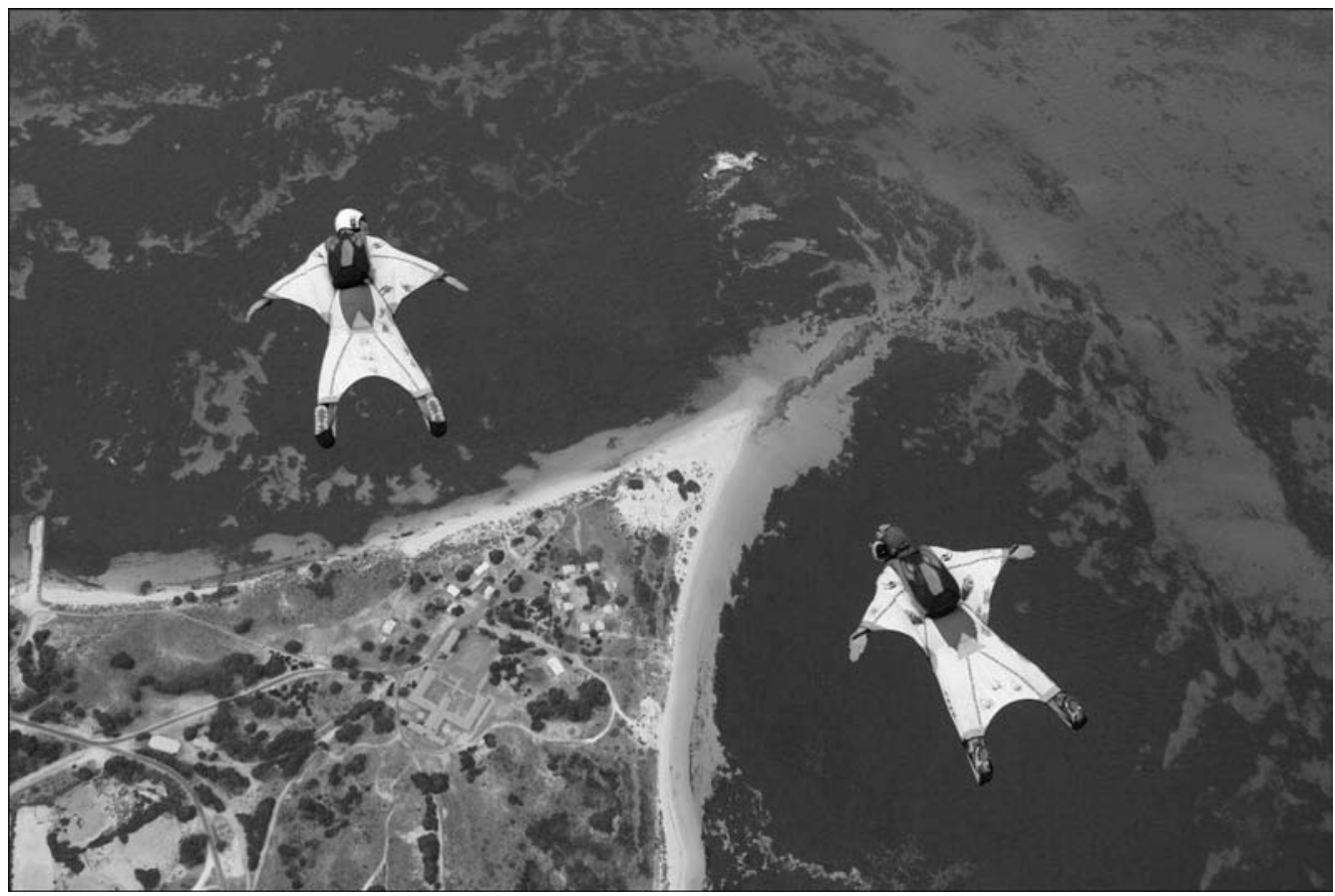

1. 2. 3. Valery Montant et Stéphane Zunino dans les wingsuits en 2005, à Perth, en Australie-Occidentale (photos de l'auteur).

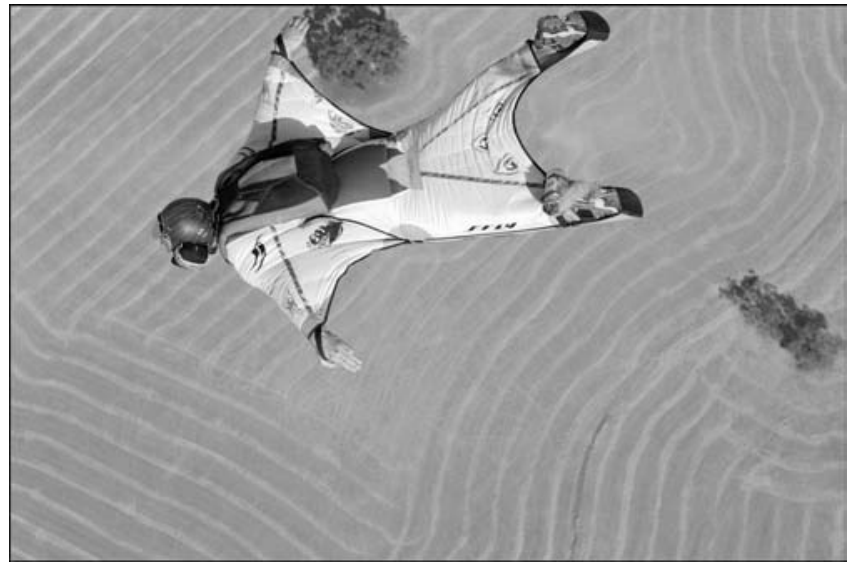

recherchent. Ce n'est pas du tout mon cas. Ressentir que je perds le contrôle est une sensation déplaisante. Dans ce cas, il n'y a plus que la chance qui joue. J'essaie justement de ne pas trop y faire appel.

Je considère que savoir qu'il existe un danger et savoir où il se trouve diminue largement celui-ci. Avec de l'expérience et une bonne technique, on peut quantifier le niveau des risques, apprécier ceux liés aux conditions météo, etc. On choisit donc dans une large mesure les risques que l'on prend!

Je n'ai pas le goût du risque, j'ai le goût de me faire plaisir... même si je sais qu'il y a un danger. Ce n'est pas le risque pour le risque, même s'il fait sûrement partie de cette attraction pour le parachutisme. Certaines

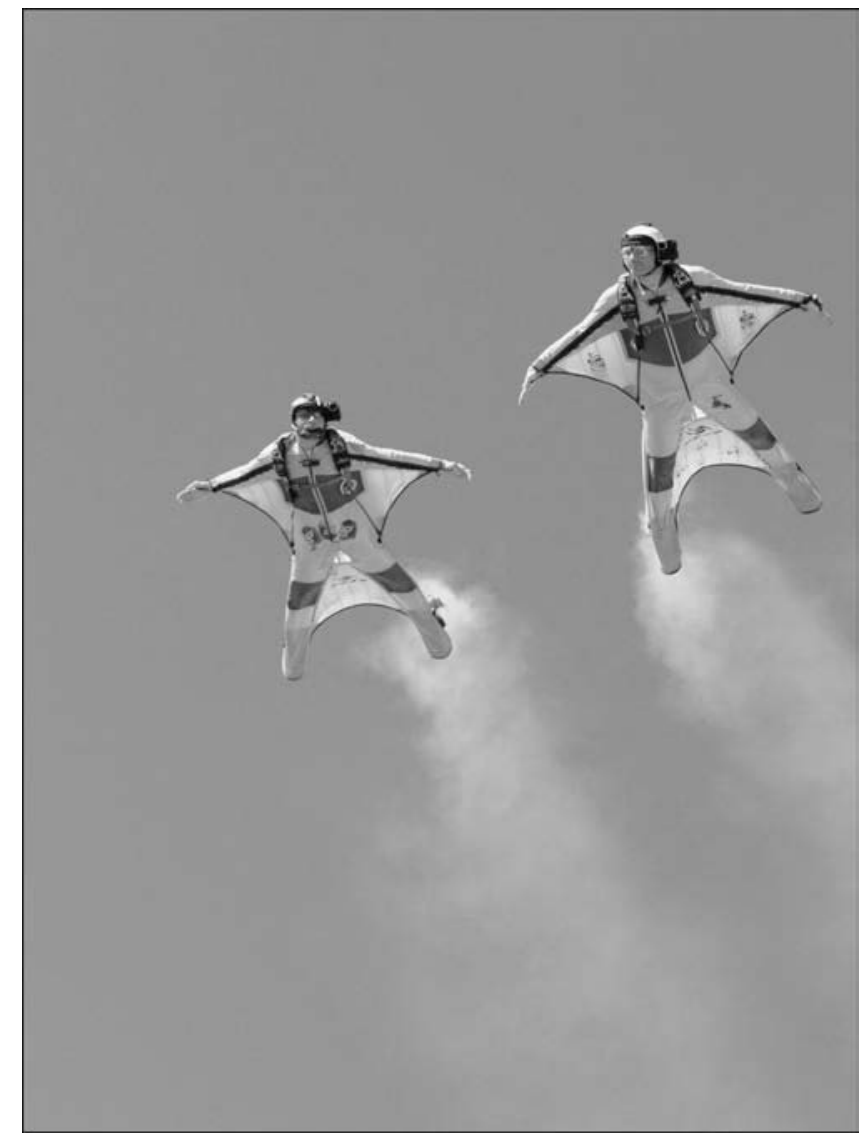


activités risquées ne m'attirent absolument pas : le saut à l'élastique ou les sports mécaniques, par exemple. Le but n'est pas le risque, mais le plaisir. Le BASE-jump en ville ne m'attire pas du tout, je ne m'y sens pas bien. Je préfere le milieu naturel. J'ai fait pas mal d'activités en mer, dans l'eau. J'ai besoin d'être dans un élément naturel puissant et d'en profiter vraiment. Ce peut être les vagues ou la puissance de l'air en chute. Le sentiment que j'éprouve quand je suis en haut d'un sommet, d'une falaise, dans le ciel est vraiment un sentiment de liberté. Mais c'est difficile d'expliquer cela.

Le risque répond à un désir de se sentir vivre. De se sentir libre. Si l'on observe bien notre société, on constate que l'on a le droit de rien faire. De nombreuses barrières viennent empêcher de prendre des risques. Elles sont principalement réglementaires. On ne peut pas boire ou fumer parce que ça donne le cancer. On ne peut pas manger parce que ça donne du cholestérol et augmente les maladies cardiovasculaires. On n'est pas autorisé à prendre des risques parce qu'on met sa vie en danger. Toutes ces activités sportives un peu extrêmes sont une possibilité pour beaucoup de s'évader et de dire : "Voilà, je fais ce que je veux. " C'est souvent un exutoire pour pas mal de gens.

On n'a plus le droit de faire grand-chose en fait. Mais que vaut la vie si elle ne peut pas être vécue ? Est-ce qu'un lion préfere vivre cinquante ans dans un zoo ou se faire éventrer par un rhinocéros au bout de vingt ans de vie sauvage? 